“( 2014 IEEE. Personal use of this material is permitted. Permission from IEEE must be obtained for all other uses, in any current or future media, including

reprinting/republishing this material for advertising or promotional purposes, creating new collective works, for resale or redistribution to servers or lists, or reuse of any copyrighted component of this work in other works." 


\title{
On Heart Rate Regulation in Cycle-Ergometer Exercise
}

\author{
Ahmadreza Argha, Steven W. Su, Sangwon Lee, Hung Nguyen, and Branko G. Celler ${ }^{\star}$, Fellow, IEEE
}

\begin{abstract}
In this paper, we have focused on the issue of regulating the human heart rate (HR) to a predefined reference trajectory, especially for cycle-ergometer exercise used for training or rehabilitation. As measuring HR is relatively easy compared to exercise intensity, it has been used in the wide range of training programs. The aim of this paper is to develop a non-model-based control strategy using proportional, integral and derivative (PID) controller/relay controller to regulate the HR to track a desired trajectory. In the case of using PID controller, the controller output signal is interpreted as a voice or auditory command, referred to as biofeedback, which can be heard by the exercising subject as a part of the controlloop. Alternatively, the relay controller output signals can be converted to some special words which can be recognised by the exerciser. However, in both cases, to effectively communicate to the user a change in exercise intensity, the timing of this feedback signal relative to the positions of the pedals becomes quite critical. A feedback signal delivered when the pedals are not in a suitable position to efficiently exert force may be ineffective and may lead to a cognitive disengagement of the user form the feedback controller. In this paper we examine the need and the consequence of synchronising the delivery of the feedback signal with an optimal and user specific placement of the pedal.
\end{abstract}

\section{INTRODUCTION}

Cycle-ergometers are normally preferable to treadmills, in terms of cost, level of noise pollution, floor space occupied and risk of falling or injury. Furthermore, during cycle-ergometer exercises, the upper body motion is usually reduced compared to the treadmill exercises, and as a result, measuring the HR is easier and more reliable [1]. Despite all these advantages for cycle-ergometer, to achieve highintensity exercise, the use of cycle-ergometers are limited to long duration exercises.

The HR can be used as an indicator to monitor the exercise intensity [2]. This makes it easier to design a control system to drive the human HR to track a predetermined and individual exercise prescription, represented as a target HR profile rather than working directly with the exercise intensity and exercise rate (ER) as control parameters.

Real-time control of the HR during treadmill exercises has been investigated using a number of different control methods, e.g. classical proportional, integral and derivative (PID) control [3], $\mathrm{H}_{\infty}$ control [4], [5] and model predictive control [6]. However, real-time control of the HR during cycle-ergometer exercises has received far less attention

A. Argha, S. W. Su, S. Lee, H. Nguyen are with Faculty of Engineering and Information Technology, University of Technology, Sydney, PO Box 123, Broadway, NSW 2007, Australia

* B. G. Celler is currently the principal scientist in the division of computational informatics, CSIRO, Marsfield, NSW 2122, Australia Branko.cellerecsiro.au to date. Broadly speaking, the problem of $\mathrm{HR}$ regulation during cycle-ergometer exercises is totally different from that of during treadmill exercises. In the treadmill case the controller system directly controls the treadmill speed and/or treadmill gradient, hence, the human does not play the role of actuator in the control-loop and they only have to passively follow the variation of the controlled parameter of treadmill speed and/or elevation. However, in the cycle-ergometer case, the controller commands are sent directly to the exercising subjects and, as a result, the human operator assumes the role of actuator of the control system. This matter is referred to as biofeedback. Note that adjusting the resistance rather than the speed of the pedaling has also been considered to change the exercise level [7]. This needs an externally controllable automatic braked cycle ergometer. But, computer controlled cycle ergometers are very expensive and are not suitable for use at home as a rehabilitation device. The air vane bike that we have selected is low cost, very sturdy and the design is particularly safe and suitable for use by frail elderly patients. Our experience is that patients find increasing workload by increasing the pedalling rate more acceptable than increasing workloads at a constant pedalling rate by increasing the braking effect. In addition the cooling effect of the air vane bike is an additional advantage.

In [8] an adaptive $\mathrm{H}_{\infty}$ controller was proposed for exerciseindependent $\mathrm{HR}$ regulation during rowing and cycling. Besides, in this reference, a periodic auditory is used to inform the subject of how hard they must work. In other words, a converter system, according to the level of controller signal, simply changes the rate of auditorys per minute to show whether more or less effort is required. However, the problem is that the auditory commands are generated without considering the position of exercising subjects' feet. One possible alteration to this audio output method might be adjusting the time duration of the auditorys and/or the frequency of auditorys rather than the rate of the auditorys per minute. This could be used to encourage the subject to apply more or less effort. Thus, in this method, the frequency of the generated auditorys/words depends on the cycling rate of the exercising subject which this itself is determined by the controller commands (see Fig. 1).

Owing to the simplicity of the structure of PID control, it is a simple and popular control strategy used in a large number of industrial and practical applications. It is well known that HR response to dynamic exercise is nonlinear and may be different for each exercising subject, in different physical situations [8]. Hence, an adaptive and model-based control method might normally be designed to address the problem of HR control. In other words, to use the traditional 


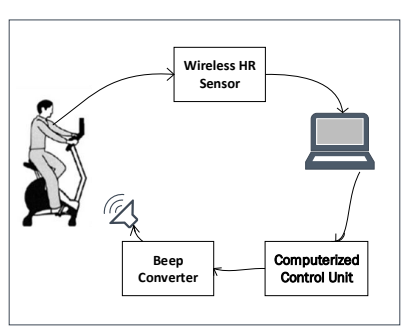

Fig. 1. Block diagram for the HR regulation system during cycling exercise

PID controller the proper settings to be used for the controller parameters should be determined continuously. Since the focus of this paper is the transduction of the controller output signal to auditory or visual command, the PID controller as a simple control strategy is used and its parameters are tuned for every exercising subject.

Furthermore, in this paper, in addition to using a PID controller to track the predetermined HR reference profile whose outputs are converted to auditory signals, a relay controller is used to generate special commands which can be read by the exerciser.

The rest of the paper is as follows: Section II shows the methodology of this paper. In Section III, controller design is discussed. Experimental results are given in Section IV. Finally, Section V concludes this paper.

\section{SYSTEM DESCRIPTION}

As mentioned, the aim of this project is to force the HR of the exercising subjects to track a predetermined prescribed profile which is subject specific and which optimises cardiorespiratory training for that patient. Fig. 1 summarises the main idea of the project. Here, the only available information from the subject is HR. Note that the specific form of activity in cycling exercise makes the use of feedback inevitable. This means that the controller commands are sent to the exerciser and it must be interpreted and implemented by the exerciser as a part of control loop. This issue motivates us to carry out some revises in the existing methods of generating biofeedback. To this end, two sensors have been installed on the pedalling system to signal when the right and left pedals are at the top of their travel. This information is used to trigger the biofeedback signal with a suitable delay to optimise the ability of the user to both interpret the command and to assert the appropriate amount of force on the pedals Furthermore, to optimise this process, we have assigned a parameter in the designed program to produce a time-delay between the actual position of the pedals and the instant that the control signals are sent. This time-delay is basically set in the program and may be adjusted to cater for individual preference, in terms of angular degree which shows the angular difference between the position of the sensor and the position where it is most effective for the exerciser to apply force to the pedal.

The sampling rate of the HR transmitted to the program by an ecg or pulse oximeter transducer is typically constant (1 sample per second), however, the rate of sending the commands to the exercising subject varies according to the pedaling rate. This form of transmitting the discrete-time signals is referred to as multi-rate transmitting technique. Note that the multi-rate sampling technique of the continuoustime signals is the counterpart of the multi-rate transmitting technique of the discrete-time signals.

\section{A. Sensors and acquisition system}

In order to collect the HR data during cycle-ergometer, in this paper, a Nonin 4100 Pulse Oximeter is used. This instrument uses a laser diode to make its measurements. Also, the air vane exercise bike selected for this project is particularly well adapted for safe exercise by the frail elderly. It has a large supporting structure that is used by the exercising patient to stabilise themselves whilst exercising. As a result, the finger pulse oximeter is almost completely stable during exercise and generates minimal artefact. An acquisition system has been designed in the National Instrument LabVIEW which provides easy synchronisation and graphic user interface.

Attached to the crank shaft of pedals on our exercise bike are two reed switches and magnets. These sensors signal a pulse whenever a full revolution has been completed. Note that according to the physical characteristics of every exerciser the suitable position for pushing the pedal is varying. Hence, in the Labview program, a time-delay parameter is designed to help the user to adjust the time-delay between the position of the pedal sensed by the sensor and the time which the command will be sent. This can be useful to adjust the point where the user can most effectively apply force to the pedals.

\section{B. HR profile}

In this project, the HR profile is selected in three stages. The first stage named the warm up period aims to increase the exerciser HR from their normal HR $\left(H R_{n}\right)$ to the exercise HR $\left(H R_{e}\right)$ which then remains constant for a predetermined time duration $\left(T_{e}\right)$. The warm up slope of exercising may be simply computed by

$$
w_{s}=\frac{H R_{n}-H R_{e}}{T_{w}},
$$

where $w_{s}$ denotes warm up slope and $T_{w}$ shows the warm up time duration. Note that this warm up time duration $T_{w}$ is determined in advance to avoid an excessively rapid increase in the HR. The second stage named exercise period is denoted by $T_{e}$. Moreover, for obtaining the exercise HR known also as ideal, target or training $\mathrm{HR}$, various formulas have been proposed so far. A commonly used formula for determining the maximum $\mathrm{HR}\left(H R_{\max }\right)$ is the well-known Haskell and Fox formula as:

$$
H R_{\text {max }}=220-\text { age. }
$$

Then, the exercising $H R_{e}$ may be set as $0.65 \sim 0.85$ of $H R_{\max }$. The third cooling down or recovery stage has a duration $\left(T_{c}\right)$ and aims to prevent possible venous pooling from an excessively abrupt termination in exercise and thus 
a reduction in HR. This duration is determined by setting the cool down slope to a suitable value.

Remark 1: In this project, the HR profile is dynamic. It means that the HR profile always starts from the subject's current resting HR and increases to a value which is calculated based on the age of the subject (aerobic training ideal HR).

\section{Exercising subjects}

In this project, 3 normal volunteers with no respiratory diseases have been used for implementing cycling exercise.

\section{CONTROllers AND CONVERTORs STRUCture}

\section{A. PID controller design}

Fig. 1 illustrates the block diagram of the control-loop of the HR regulation system using PID controller. The discrete-time PID controller is derived from the traditional continuous-time PID controller using its velocity form expressed in a difference equation form. Because of the difference equation form and lack of the integral action the problem of integral windup is avoided. This controller is as follows,

$$
\begin{aligned}
u(k)=u(k-1) & +K_{P}[e(k)-e(k-1)]+K_{I} e(k) \\
& +K_{D}[e(k)-2 e(k-1)+e(k-2)],
\end{aligned}
$$

where

$$
e(k)=r(k)-y(k),
$$

in which $u(k), r(k), y(k), K_{P}, K_{I}$ and $K_{D}$ are control effort, reference $\mathrm{HR}$, actual $\mathrm{HR}$ (all at $k$ th instant), proportional gain, integral gain and derivative gain, respectively. As seen, the control effort at $(k-1)$ th instant is used to obtain the control effort at $k$ th instant. Furthermore, different heuristic methods have been developed for the tuning of the PID parameters. By far, the best known is the Ziger-Nichols technique.

\section{B. Auditory convertor}

In this project, the output signal of the PID controller is converted to a sequence of auditory signals to inform the subject of how hard and when they must exert force on the pedals. A converter system, proportional to the level of the controller signal, changes both the time duration of auditory signal as well as its frequency to indicate whether more or less effort is required. The combination of varying the time duration and the frequency is found to be more effective in facilitating the user to apply more or less effort.

Various techniques for changing in auditory signals' characteristics have been tested to find an appropriate characteristic of the auditory signal (such as time duration and frequency) which can be best interpreted by the exercisers to change the intensity and timing of their activity. The outcome of the investigation was a protocol which changes both frequency and duration of the acoustic feedback signal, simultaneously. This means that for the slow pedalling a longer time duration and lower frequency may be more effective and vice versa at high speed. Furthermore, note that there are a number of limitations for the range of time duration and frequency which can be used. For instance, if the time duration is more than a specific value the socalled aliasing phenomena may happen and, time durations which are less than a specific value can not be heard by the users. This issue also exists for the frequency of the auditory signals. Eventually, the best range of time duration and frequency of the auditorys was selected as $60 \sim 500 \mathrm{~ms}$ and $500 \sim 2000 \mathrm{~Hz}$, respectively. Hence, the converter effectively implements two saturation functions,

$$
t_{d}=\operatorname{sat}\{g(u(k))\}= \begin{cases}60 & g(u(k)) \leq 60 \\ g(u(k)) & 60<g(u(k))<500 \\ 500 & g(u(k)) \geq 500\end{cases}
$$

where $t_{d}$ denotes the time duration of the auditory signals and $g(u(k))$ is a scaling function which converts the range of $u(k)$ to the range of time duration, and,

$f_{b}=\operatorname{sat}\{h(u(k))\}= \begin{cases}500 & h(u(k)) \leq 500 \\ h(u(k)) & 500<h(u(k))<2000 \\ 2000 & h(u(k))>2000,\end{cases}$

where $f_{b}$ denotes the frequency of the auditory signals and $h(u(k))$ is a scaling function which converts the range of $u(k)$ to the range of appropriate frequencies of the auditory signal.

Remark 2: It should be noted that, in fact, the above system includes two distinct control loops. In simpler terms, the explained control system is a MISO (2I1O) in which the PID controller of the both control loops have the same parameters.

\section{Relay controller Strategy}

This section explains the details of implementation of the relay controller designed for the tracking the HR profile during the cycling. This controller generates a sequence of commands by showing a number of words on the computer station. Every word, in fact, implies the desired action required by the subject to achieve the desired HR. The commands are determined based on the error defined in (4). The switching of the commands are as:

$$
u(k+1)=\left\{\begin{array}{lr}
\text { Highly speedup } & e(k)>\beta \\
\text { Slightly speedup } & \alpha<e(k) \leq \beta \\
\text { Maintain } & -\alpha \leq e(k) \leq \alpha \\
\text { Slightly slowdown } & -\beta \leq e(k)<-\alpha \\
\text { Highly slowdown } & e(k)<-\beta
\end{array}\right.
$$

Similar to the PID control part, again, the relay controller commands are synchronised to the position of the pedals which are sensed via the reed sensors. In other words, the control commands are multi-rate and their rate depends on the rate of pedalling.

\section{EXPERIMENTAL RESULTS}

This section will describe the results and performance of controllers designed for this project. As mentioned, this project has been carried out on three healthy male subjects 


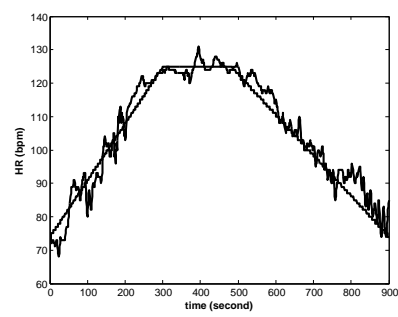

Fig. 2. HR profile tracking during cycling using PID controller

during cycling exercise. The HR profile was defined in 3 stages; warm up, exercise and cool down, where each stage lasted 5, 3 and 7 minutes, respectively. Moreover, the reference value of $125 \mathrm{bpm}$ is our desired aerobic training HR for all subjects during the exercise stage.

The PID controller parameters have been tuned to $K_{P}=5$, $K_{I}=0.3$ and $K_{D}=0.02$. The tracking performance of this controller for one subject is demonstrated in Fig. 2. It can be concluded from this result that the designed PID controller can efficiently drive the subject to track the desired HR profile.

In addition, as another simple control strategy, we have used the relay controller explained in Section III-C, with the following tuned parameters:

$$
\alpha=3, \beta=10 .
$$

The performance of this control strategy is shown in Fig. 3. This figure implies that the relay control method can effectively help the user to track the desired HR profile. However, clearly, the performance of the PID control is more acceptable compared to the relay controller. Broadly speaking, the relay controller is a discontinuous controller which works based on switching functions. Note that the parameters $\alpha$ and $\beta$ in (7) play important role in the performance of this controller. Smaller values of these parameters may lead to better performance. However, meanwhile, it can result in more visual commands (words on the computer station) which subject has to read continuously. This may contribute to cognitive overload and disengagement for some subjects.

It should be mentioned that in the case that exercising subject inadvertently or deliberately choose not to follow the controller commands, a big tracking error will be generated, and the controller will require a huge effort to compensate the backwardness. This may result in a big fluctuation in the HR of the subjects. In order to address this problem the HR profile needs to be more dynamic. In other words, in the cases that big tracking errors are noted, the HR profile should be adaptively revised to prevent big efforts of the subjects. This might be the subject of our future works.

\section{CONCLUSiOns}

In this study, an automated system is designed to help exercising subjects to track a predetermined HR profile. Two kind of control methods (PID controller and relay controller), which are simple to design and implement, are deployed

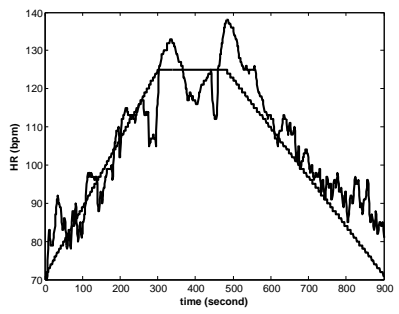

Fig. 3. HR profile tracking during cycling using Relay controller

in this project. The significance of this study, compared to the previous works, is this fact that the biofeedback signals are synchronised with respect to the positions of the pedals. To this end, for the PID controller case, by adjusting the time duration and the frequency of the auditory signals rather than the rate of the auditory signals per minute the HR of the subjects are forced to track the profile. Accordingly, this system is basically converted to a MISO (2I1O) control system. In addition to PID controller, a simple relay controller is also used to compare its performance with PID controller. Experimental results which have been carried out on the three male subjects, validate the efficiency of the system. Furthermore, it has been found that the PID controller has better performance in terms of less oscillations of the subject's HR. A dynamic HR profile designing method has been considered for further study.

\section{REFERENCES}

[1] G.F. Fletcher, G.J. Balady, E.A. Amsterdam, B. Chaitman, R. Eckel, J. Fleg, V.F. Froelicher, A.S. Leon, I.L. Piña R. Rodney, D.A. Simons Morton, M.A. Williams and T. Bazzarre, Exercise standards for testing and training: A statement for healthcare professionals from the American Heart Association, Circulation, vol. 104, pp 1694-1740, 2001.

[2] M. Paradiso, S. Pietrosanti, S. Scalzi, P. Tomei and C.M. Verrelli, Experimental Heart Rate Regulation in Cycle-Ergometer Exercises, IEEE Transactions on biomedical engineering, vol. 60, no. 1, pp 135139, 2013.

[3] T. Kawada, G. Sunagawa, H. Takaki, T. Shishido, H. Miyano, Miyashita, T. Sato, M. Sugimachi and K. Sunagawa, Development of a servo-controller of heart rate using a treadmill, Japanese Circulation Journal, vol. 63, pp 945-950, 1999.

[4] T.M. Cheng, A.V. Savkin, B.G. Celler, S.W. Su, and L. Wang, Nonlinear modeling and control of human heart rate response during exercise with various work load intensities, IEEE Transactions on biomedical engineering, vol. 55, no. 11, pp 2499-2508, 2008.

[5] S.W. Su, L. Wang, B.G. Celler, A.V. Savkin, and Y. Guo, Identification and control for heart rate regulation during treadmill exercise, IEEE Transactions on biomedical engineering, vol. 54, no. 7, pp. 1238-1246, 2007.

[6] S.W. Su, S. Huang, L. Wang, B.G. Celler, A.V. Savkin, Y. Guo and T. Cheng, Nonparametric hammerstein model based model predictive control for heart rate regulation, Conf. Proc. IEEE Eng. Med. Biol. Soc., pp 2984-2987, 2007.

[7] T. Kawada et al, Development of a servo-controller of heart rate using a cycle ergometer, Heart Vessels, no. 14, pp 177-184, 1999.

[8] D. Baig, F. Javed, A.V. Savkin and B.G. Celler, An Adaptive $\mathrm{H}_{\infty}$ Control Design for Exercise-Independent Human Heart Rate Regulation System, 9th IEEE International Conference on Control and Automation (ICCA), Santiago, Chile, Dec. 19-21, 2011

[9] K. Weng, B. Turk, L. Dolores, T.N. Nguyen, B.G. Celler, S.W. Su and H.T. Nguyen, Fast tracking of a given heart rate profile in treadmill exercise, 32nd Annual International Conference of the IEEE EMBS, Buenos Aires, Argentina, August 31 - September 4, 2010. 